\title{
Vertrauen und Führung im Kontext digitaler Arbeit
}

\author{
Ariane Jäckel ${ }^{1}$ \\ Online publiziert: 16. April 2020 \\ (c) Der/die Autor(en) 2020
}

\section{Zusammenfassung}

Der vorliegende Beitrag der Zeitschrift „Gruppe. Interaktion. Organisation. (GIO)“ untersucht die Relevanz von Vertrauen zwischen Führungskraft und Mitarbeiter im Kontext der Digitalisierung und kommt zu dem Ergebnis, dass Vertrauen in Zukunft noch bedeutsamer wird. Der Beitrag gelangt zu dieser Erkenntnis, indem er die psycho-sozialen Auswirkungen der Digitalisierung auf organisationaler Ebene identifiziert und systematisch die möglichen Wirkzusammenhänge zu Vertrauen analysiert. Die Analyse ergibt, dass sich die Zahl der Anwendungsfelder für Vertrauen im Kontext der Digitalisierung deutlich erhöht, Machtausübung als alternatives Konzept an Potenzial verliert und Kontrolle die Funktion von Vertrauen sowie dessen positive Auswirkungen nicht ersetzen, aber ergänzen kann. Spezifische Implikationen zum Aufbau von Vertrauensbeziehungen im Kontext digitaler Arbeitswelten schließen diesen Beitrag ab.

Schlüsselwörter Arbeit 4.0 · Digitalisierung $\cdot$ Führung $\cdot$ Kontrolle $\cdot$ Macht $\cdot$ Vertrauen

\section{Trust and leadership in the context of digital work}

\begin{abstract}
This contribution to the journal "Gruppe. Interaktion. Organisation. (GIO)" investigates the relevance of trust between leaders and followers in the context of digitalization and comes to the conclusion that trust is getting even more important. The article reaches this conclusion by firstly identifying the psycho-social effects of digital work on an organizational level and secondly analyzing the possible interactions with trust. The analysis demonstrates that in the context of digitalization the application fields of trust are increasing significantly, whereas the potential of the alternative concept of power is decreasing while the concept of control can complement trust but cannot substitute the function and positive outcomes that trust has. The article ends with specific implications for the development of trust in the context of digital work.
\end{abstract}

Keywords Control · Digitalization · Leadership · New work · Power · Trust

\section{Einleitung}

Science-Fiction oder Realität? Vor kurzem wurde bekannt, dass sich Mitarbeiter von TUI Nordic einen Mikrochip implantieren lassen können, mit dem sie in der Lage sind, z.B. Türen zu öffnen oder den hauseigenen Snackautomaten zu bedienen (vgl. Läsker 2019). Nur ein Beispiel für die digitale Transformation der Arbeit. In ihr kumulieren die Megatrends der heutigen Zeit. Organisationen werden

Dr. Ariane Jäckel

uni@ariane-jaeckel.de

1 Fachgebiet Arbeits- und Organisationspsychologie, Universität Kassel, Kassel, Deutschland u. a. durch neue Interaktionsformen, schnelleres und flexibleres Arbeiten sowie globale Kooperationen herausgefordert. Sich erfolgreich diesen Herausforderungen zu stellen, ist eine zentrale Gelingensbedingung organisationaler Führung. Als eine Schlüsselkomponente für diesen Erfolg wird wie selbstverständlich immer wieder Vertrauen genannt (vgl. z. B. Diekhöner 2018; Schönfelder 2019). Wenn jedoch selbst der Snackautomat weiß, wann der Mitarbeiter während der Arbeit welchen Schokoriegel aus ihm herausholt, stellt sich die Frage, in welchem Ausmaß Vertrauen in der Beziehung zwischen Führungskraft und Mitarbeiter für erfolgreiches organisationales Handeln in einer digitalisierten Arbeitswelt noch relevant ist.

Betrachtet man die bereits vorhandene Literatur zu dieser Fragestellung bzw. zum Thema Vertrauen und Digitalisie- 
Abb. 1 Psycho-Soziale Auswirkungen der digitalen Transformation von Arbeit

\begin{tabular}{|c|c|l|}
\hline \multirow{2}{*}{$\begin{array}{c}\text { Einsatz digitaler } \\
\text { Technik in } \\
\text { Organisationen }\end{array}$} & \multirow{2}{*}{$\begin{array}{c}\text { Auswirkungen } \\
\text { auf psycho- } \\
\text { sozialer Ebene }\end{array}$} & Komplexitätserweiterung \\
\cline { 3 - 3 } & & Veränderung von Strukturen und Prozessen \\
\cline { 3 - 3 } & & Räumliche und zeitliche Ungebundenheit \\
\cline { 3 - 4 } & &
\end{tabular}

rung, so hat man zumeist die Wahl zwischen oberflächlich postulierten Zusammenhängen, z.B. „Was ist zu tun? Es ist ein Wandel hin zu einer offenen Unternehmenskultur zu schaffen, die von Vertrauen, Austausch und Kooperation getragen ist" (Schmiech 2018, S. 22), sowie einigen konkreten Erkenntnissen, die sich jedoch auf sehr spezifische Aspekte der Thematik beziehen, z. B. „Die Effektivität von virtuellen Teams hängt demnach in größerem Umfang von Vertrauen ab“ (Werther et al. 2018, S. 54). Der vorliegende Artikel liefert einen Beitrag, um die dazwischen liegende Lücke konzeptionell zu schließen. Zu diesem Zweck wird zunächst ein systematischer Überblick über die möglichen Wirkzusammenhänge zwischen Vertrauen und Digitalisierung erarbeitet, um davon ausgehend die Relevanz von Vertrauen zu beurteilen und konkrete Handlungsempfehlungen für die organisationale Praxis abzuleiten.

\section{Aspekte einer digitalisierten Arbeitswelt}

Die Digitalisierung der Arbeitswelt wird in Deutschland insbesondere unter der Bezeichnung Arbeit 4.0 behandelt (vgl. Bundesministerium für Arbeit und Soziales 2016). Es handelt sich jedoch um kein festes Konzept, sondern eher um einen Sammelbegriff für die „Beschreibung zukünftiger Arbeitswelten, die von zunehmender Digitalisierung, Globalisierung und Individualisierung geprägt sind“ (Bruckner und Werther 2018, S. 17). Diese zukünftige Arbeitswelt umfasst sowohl die verstärkte Nutzung digitaler Arbeitsmittel (E-Mail, Smartphones, Apps und Tablets sowie Robotik) als auch veränderte Formen der Zusammenarbeit im Betrieb (z.B. Homeoffice, arbeiten von unterwegs, virtuelle Besprechungen) (vgl. Ahlers 2018). Der vorliegende Beitrag schlägt die in Abb. 1 dargestellte Konzeptualisierung vor, um die digitale Transformation der Arbeitswelt auf der vertrauensrelevanten psycho-sozialen Ebene zu erfassen.

Diese Konzeptualisierung lässt sich wie folgt erläutern: In den Aufzählungen der digitalen Techniken, die das Arbeiten bereits jetzt und in naher Zukunft verändern, besteht große Einigkeit, wenn man von vagen Zukunftsvisionen absieht: Big-Data, Cloud-Computing, Mobile Devices, neue Kommunikationskanäle, Internet der Dinge, Chipping, Robotik, Künstliche Intelligenz, Augmented Reality, E-Learning etc. (vgl. z. B. Ahlers 2018; Schaff 2019). Diese Techniken werden in Organisationen primär eigesetzt, um ökonomisch wertvolle Funktionen zu erfüllen, wie z.B. Pro- duktivitätssteigerung, Arbeitsentlastung, größere Netzwerke, verbesserte Organisation und Kommunikation, Wissenstransfer. Hierdurch wird ein Arbeitskontext generiert, der Auswirkungen auf die Psyche des Einzelnen sowie die soziale Interaktion in Organisationen hat (vgl. Abb. 1). Die für das Vertrauensphänomen relevanten psycho-sozialen Auswirkungen lassen sich auf Basis bereits bestehender Ansätze (vgl. z. B. Hirsch-Kreinsen 2015; Werther et al. 2018) wie folgt zusammenfassend benennen:

- Komplexitätserweiterung,

- Veränderung von Strukturen und Prozessen,

- räumliche und zeitliche Ungebundenheit sowie

- flache Hierarchien.

Was diese Auswirkungen für Führungskräfte und Mitarbeiter bedeuten und welche Wirkzusammenhänge zu Vertrauen bestehen, ist Gegenstand der folgenden Ausführungen.

\section{Vertrauen zwischen Führungskraft und Mitarbeiter im Kontext der Digitalisierung}

Vertrauen ist ein Phänomen, das ubiquitär relevant und gleichzeitig schwer fassbar ist (vgl. Endress 2002). Daher wird es in der Praxis und in der Wissenschaft zu Unrecht häufig synonym zu Begriffen wie Vertrautheit, $\mathrm{Zu}$ versicht, Voraussehbarkeit oder Vertrauenswürdigkeit verwendet (vgl. Geramanis 2001; Mayer et al. 1995). Dabei formuliert die organisationale Vertrauensforschung inzwischen einen klar umrissenes Konzept (vgl. Möllering et al. 2004): Bei Vertrauen handelt es sich um eine spezifische positive Erwartung in den Vertrauensnehmer, die auf einer kontingenzbewältigenden Komplexitätsreduktion (,leap of trust“ Möllering 2001, p. 413) basiert, aus der heraus dann schließlich eine handlungsbefähigende Bereitschaft sich verletzlich zu machen resultiert (vgl. Luhmann 2000 [1968]; Mayer et al. 1995). So hat z. B. eine vertrauende Führungskraft die sichere Erwartungshaltung, dass ihr Mitarbeiter in ihrem Sinne handelt (obwohl vieles anderes möglich wäre), und kann auf dieser sicheren Erwartung ihr eigenes Handeln und das Handeln anderer, denen sie ebenfalls vertraut, aufbauen lassen.

Die Besonderheiten der Vertrauensbeziehung zwischen Führungskraft und Mitarbeiter liegen darin begründet, dass diese in einem organisationalen Rahmen und mit einem 
unterschiedlich stark ausgeprägten hierarchischen Gefälle, durch das die Führungskraft Macht über den Mitarbeiter erhält, stattfindet (vgl. Jäckel 2018). Sowohl aus Führungskraft- als auch aus Mitarbeiterperspektive ist Vertrauen ein wichtiger Bestandteil dieser organisationalen Beziehung und erfüllt für beide Parteien wichtige Funktionen. Das Vertrauen des Mitarbeiters in die Führungskraft steht z.B. in einem engen Zusammenhang mit der Arbeitszufriedenheit des Mitarbeiters (vgl. Dirks und Ferrin 2002). Es fördert das Gefühl der Sicherheit in Bezug auf die Arbeit sowie den Arbeitsplatz und erhöht gleichzeitig das Autonomieerleben des Mitarbeiters (vgl. Jäckel 2018). Aus der Sicht der Führungskraft ermöglicht Vertrauen insbesondere die Koordination komplexer organisatorischer Handlungen, zu denen diese ohne eine sichere positive Erwartungshaltung an ihre Mitarbeiter nicht imstande wäre (vgl. Bachmann 2003). Auch ein positiver Zusammenhang zur Arbeitsleistung, zum Organizational-Citizenship-Behavior (OCB) und zur Selbstwirksamkeit sowie eine verbesserte Kommunikation und der Transfer von Wissen werden durch Studien hinreichend belegt (vgl. Burke et al. 2007; Dirks und Ferrin 2002).

Vertrauen konkurriert in der Beziehung zwischen Führungskraft und Mitarbeiter aus Sicht der Führungskraft mit der Ausübung hierarchisch bedingter Macht und Kontrolle. Sowohl Macht als auch Kontrolle können auf unterschiedliche Weise ebenfalls dazu genutzt werden, eine sichere Grundlage für organisatorisches Handeln zu erlangen (vgl. Bachmann 2003; Mayer et al. 1995).

\subsection{Wirkzusammenhänge zwischen Vertrauen und den Auswirkungen der Digitalisierung in Organisationen}

Im Folgenden werden die möglichen Wirkzusammenhänge des Vertrauens zwischen Führungskraft und Mitarbeiter und den Auswirkungen der Digitalisierung auf psycho-sozialer Ebene analysiert (vgl. Abb. 2). Inwieweit Macht und Kontrolle hierbei eine Alternative zu Vertrauen darstellen, wird im nächsten Abschnitt (vgl. Abschn. 3.2) diskutiert.

Komplexitätserweiterung $\mathrm{Zu}$ den wesentlichen Folgen der Digitalisierung zählt die erweiterte Komplexität von Arbeit (vgl. Kap. 2). Dieser Umstand bedarf jedoch der Klärung, da digitale Technik unter anderem das Gegenteil bewirken und Arbeitsvorgänge erleichtern und vereinfachen soll. Ein gutes Beispiel hierfür ist die Möglichkeit, mit wenigen Eingaben von überall und zu jeder Zeit mit seinem Tablet oder Smartphone wichtige Daten über das Internet abzurufen, zu senden und sich zu vernetzen. Dieser Vorgang an sich bedeutet zunächst einen Gewinn an Flexibilität sowie eine Arbeits- und Zeitersparnis. Die Zeitersparnis bedeutet jedoch in einem ökonomischen Kontext zumeist nicht, dass Führungskräfte und Mitarbeiter hierdurch mehr Zeit haben, sondern dass in der gleichen Zeit mehr gearbeitet werden kann. Zudem entstehen durch die Technik vielfältige neue Möglichkeiten des Arbeitens, aus denen die sinnvollste ausgewählt werden muss (vgl. Kap. 2). Wie die Ausführungen verdeutlichen, entsteht die Komplexität, die mit der Digitalisierung einhergeht, durch Akzeleration und Vervielfältigung der Möglichkeiten. Sie führt letztlich da$\mathrm{zu}$, dass ein einzelner Mensch organisationale Prozesse und Strukturen nicht mehr als Ganzes detailliert erfassen kann. Sowohl Führungskraft als auch Mitarbeiter stehen vor der Herausforderung damit umzugehen. Insbesondere die Führungskraft steht vor der Herausforderung, dass sie komplexe organisatorische Handlungen bzw. Prozesse nur dann koordinieren kann, wenn sie sich auf ihre Mitarbeiter verlassen kann bzw. sie davon ausgehen kann, dass ihre Erwartungen nicht enttäuscht werden. Ein Beispiel hierfür ist das Durchführen von Projekten in einem akzelerierten und flexibilisierten digitalen Arbeitskontext, die von der Führungskraft nur geplant und realisiert werden können, wenn die positive Erwartung besteht, dass die Mitarbeiter die komplexen, ineinandergreifenden bzw. voneinander abhängigen Arbeitspakete planmäßig umsetzen. Vertrauen leistet genau das. Es führt zu positiven Erwartungen in das Verhalten anderer und ermöglicht so die Koordination und Umsetzung komplexer Handlungen, die von der Verwirklichung aufeinander aufbauender Erwartungen in andere abhängig ist (vgl. Bachmann 2003).

Veränderung von Strukturen und Prozessen Die Digitalisierung der Arbeit ist ein Prozess, der viele, unterschiedlich tief greifende Veränderungen in Organisationen hervorruft. Das fängt bei mobilen Endgeräten, veränderten Kommunikationskanälen sowie Vernetzung an und reicht bis zu vollautomatisierten Prozessen oder Mitarbeitern, bei denen, wie im eingangs erwähnten Beispiel (vgl. Kap. 1), Mikrochips implantiert werden. Diese Veränderungen, die organisationale Verbesserungen herbeiführen sollen, rufen jedoch nicht nur Begeisterung, sondern auch Unsicherhei-

\begin{tabular}{|c|c|c|c|c|}
\hline \multirow{4}{*}{$\begin{array}{c}\text { Einsatz digitaler } \\
\text { Technik in } \\
\text { Organisationen }\end{array}$} & \multirow{4}{*}{$\begin{array}{c}\text { Auswirkungen } \\
\text { auf psycho- } \\
\text { sozialer Ebene }\end{array}$} & Komplexitätserweiterung & \multirow{4}{*}{ Vertrauen } & Positive Erwartung \\
\hline & & Veränderung von Strukturen und Prozessen & & \\
\hline & & Räumliche und zeitliche Ungebundenheit & & котріexıtatsrequкпоо \\
\hline & & Flache Hierarchien & & Risiko-Bereitschaft \\
\hline
\end{tabular}

Abb. 2 Mögliche Wirkzusammenhänge von Vertrauen im Kontext digitaler Arbeit 
ten und Widerstände bei den Mitarbeitern hervor, die sich z.B. übergangen und überfordert fühlen, Angst um ihre Freiheiten oder sogar Angst um ihre Arbeitsstelle haben. „Um mit dieser Unsicherheit besser umgehen zu können, benötigen Mitarbeiterinnen und Mitarbeiter mehr denn je Führungskräfte, auf die sie sowohl persönlich als auch fachlich zählen können. MitarbeiterInnen müssen ihren Führungskräften vertrauen können“ (Malczok und Kirchhoff 2019, S. 223). Es ist keine neue Erkenntnis, dass Vertrauen eine wichtige Rolle für Veränderungsprozesse spielt (vgl. Hoe 2007). Die Begründung hierfür ist, dass sowohl Führungskraft als auch Mitarbeiter während des Veränderungsprozesses Risiken eingehen müssen, damit dieser schlussendlich gelingen und nachhaltig implementiert werden kann. Vertrauen ermöglicht das Eingehen dieser Risiken. Mitarbeiter vertrauen z. B. ihrer Führungskraft, dass die Automatisierung bestimmter Arbeitsabläufe nicht dazu führt, dass die Mitarbeiter ihren Arbeitsplatz verlieren oder durch das Sammeln von Daten (z.B. durch Leistungs- und Zeiterfassungssysteme, Messenger-Nachrichten, GPS-Daten) ihre Persönlichkeitsrechte verletzt werden. Führungskräfte vertrauen umgekehrt ihren Mitarbeitern, dass diese den Veränderungsprozess mit all ihrer Kompetenz unterstützen. Hierzu gehört auch der Transfer von Wissen, der ein wesentlicher Bestandteil organisationalen Lernens und eines jeden Veränderungsprozesses ist (vgl. Hoe 2007). Vertrauen zwischen Führungskraft und Mitarbeiter beseitigt Unsicherheiten und befördert somit das Gelingen von Veränderungsprozessen, die im Kontext der beständig voranschreitenden Digitalisierung nicht mehr die Ausnahme, sondern vielmehr die Regel im organisationalen Arbeitsalltag darstellen.

Räumliche und zeitliche Ungebundenheit Ein weiterer bedeutsamer Aspekt, wie die Digitalisierung schon jetzt unseren Arbeitsalltag verändert, ist die räumliche und zeitliche Ungebundenheit bzw. Flexibilisierung von Arbeit, die von einigen, mit negativer Konnotation, auch als räumliche und zeitliche Entgrenzung bezeichnet wird (vgl. Hirsch-Kreinsen 2015; Werther et al. 2018). Es ist in vielen Fällen nicht mehr nötig und gegebenenfalls auch nicht effizient, dass alle Mitarbeiter sich zu einer bestimmten Zeit an einem bestimmten Ort versammeln, um ihrer Arbeit nachzugehen. Gleiches gilt nicht nur für die zu verrichtende Arbeit, sondern auch für die gemeinsame Kommunikation. Dies hat viele Vorteile, doch es ist offensichtlich, dass sowohl Mitarbeiter als auch insbesondere Führungskräfte hierbei Risiken eingehen, damit diese Form der freien Arbeit funktioniert. Beispiele hierfür sind die Konzepte des Homeoffice und der Vertrauensarbeitszeit, bei denen die Führungskraft das Risiko eingeht, dass der Mitarbeiter nicht so lange und fokussiert wie erwartet arbeitet, und der Mitarbeiter befürchten muss, dass seine Arbeit nicht wahrgenommen und wertge- schätzt wird. Wenn Führungskraft und Mitarbeiter jedoch einander vertrauen, tritt eine sichere, positive Erwartungshaltung an die Stelle der zuvor bestehenden Kontingenz, auf deren Basis die mit Risiken versehene freie Arbeitsform erfolgreich realisiert werden kann. Vertrauen kann somit entscheidend zum Eingehen der Risiken, die mit einer räumlich und zeitlich ungebundenen Arbeit einhergehen, beitragen (vgl. Deutsche Gesellschaft für Personalführung 2016). Allerdings erzeugen die räumliche Distanz und die damit verbundene Kommunikation über digitale Kanäle auch Barrieren für den Vertrauensaufbau (vgl. Kauffeld et al. 2016); denn entscheidend für den Aufbau von Vertrauen ist die Vertrauenswürdigkeit einer Person, die durch die Zuschreibung von Kompetenz, Wohlwollen und Integrität entsteht (vgl. Mayer et al. 1995). Um diese drei Eigenschaften zu attribuieren, werden jedoch Informationen über den Vertrauensnehmer benötigt, welche in persönlicher Face-To-FaceInteraktion deutlich einfacher gewonnen werden können als über digitale Kommunikation, bei der immer Informationen über Person und Kontext durch die Beschränkungen des jeweiligen Kommunikationskanals verlorengehen.

Flache Hierarchien Vor dem Hintergrund digitaler Transformation wird die Verflachung von Organisationsstrukturen gefördert und der hierarchische Unterschied zwischen Führungskraft und Mitarbeiter wird zunehmend nivelliert (vgl. z. B. Depiereux und van Alphen 2018). Dies lässt sich zum einen damit begründen, dass Organisationen versuchen Hierarchien abzubauen, da ihnen ansonsten die notwendige Agilität in digitalen Märkten fehlt. Zum anderen führt die Digitalisierung dazu, dass Informationsprivilegien verlorengehen, die in einer hierarchischen Organisation zur Legitimation von Führung beitragen. Somit ist der einzelne Mitarbeiter deutlich besser informiert und gleichzeitig zu autonomeren Handlungen befähigt (vgl. Kruse und Schomburg 2016). Diese flachen Hierarchien, denen eine Organisationskultur der Kooperation und Gleichberechtigung zugrunde liegt, begünstigen den Aufbau von Vertrauensbeziehungen zwischen Führungskraft und Mitarbeiter. Hierfür lassen sich zwei Ursachen benennen: zum einen erscheinen für den Mitarbeiter die Risiken in der Beziehung zwischen Führungskraft und Mitarbeiter aufgrund des größeren Autonomieerlebens geringer, was den Einstieg in den Vertrauensaufbau erleichtert, und zum anderen verfügen Führungskräfte über weniger Alternativen zu Vertrauen, da ihre Macht schwächer ist als in Organisationen mit ausgeprägten Hierarchien (vgl. Bachmann 2003). Dies hat zur Folge, dass Autorität und Macht einer Zusammenarbeit weichen, „die auf Vertrauen und Kooperation basiert“" (Werther et al. 2018, S. 48). 


\subsection{Macht und Kontrolle - Mögliche Alternativen zu Vertrauen in einer digitalen Arbeitswelt?}

Die Analyse der oben stehenden Wirkzusammenhänge verdeutlicht, dass Vertrauen ein bedeutsames Potenzial besitzt, um die Herausforderungen der Digitalisierung zu bewältigen. Dies bedeutet in der Folge jedoch nicht zwingend, dass es neben Vertrauen keine alternativen Konzepte gibt, die für eine Führungskraft eine vergleichbare oder eventuell noch bessere Wahl bieten. Eine bislang unerwähnte Problematik von Vertrauen besteht schließlich darin, dass es enttäuscht werden kann und auf diese Weise sowohl auf der persönlichen als auch auf der organisatorischen Ebene schwere Schäden entstehen können (vgl. Skinner et al. 2014). Zur „dark-side of trust“ (Gargiulo und Ertug 2006) gehört zudem das Phänomen des blinden Vertrauens, bei welchem in einem zu hohen Ausmaß vertraut wird, was dann z.B. zu irrationalen Entscheidungen und mangelnder Innovationsfähigkeit führen kann (vgl. Molina-Morales et al. 2011). Ebenso muss noch der anfängliche Aufwand für den Aufbau einer Vertrauensbeziehung berücksichtigt werden, der durch die räumliche und zeitliche Ungebundenheit von Arbeit sogar noch größer wird (vgl. Abschn. 3.1).

Als Alternative stehen durch ihre Funktion das Ausüben von Macht und Kontrolle in Konkurrenz zu Vertrauen. Macht kann im organisationalen Kontext definiert werden als das Vermögen, andere Parteien durch die Kontrolle von Ressourcen (z.B. Sanktion, Legitimation, Information) zu beeinflussen (vgl. Raven et al. 1998). Machtausübung funktioniert auf ähnliche Weise wie Vertrauen, jedoch mit dem Unterschied, dass sich die positiven Erwartungen nicht aus der Vertrauenswürdigkeit des Mitarbeiters ergeben, sondern aus den negativen Sanktionen, die dem Mitarbeiter bei Nicht-Erfüllung der Erwartung drohen (vgl. Bachmann 2003; Jäckel 2018; von Ameln und Kramer 2012). Im Kontext der Digitalisierung wird Machtausübung als Alternative zu Vertrauen jedoch dadurch geschwächt, dass die digitale Umgestaltung von Organisationen wie bereits erwähnt hierarchische Unterschiede zunehmend nivelliert (vgl. Abschn. 3.1). Flache Hierarchien bedeuten weniger Macht für die Führungskräfte einer Organisation und somit auch weniger Möglichkeiten, um mittels Machtausübung Komplexität zu reduzieren und Erwartungen erfolgreich zu koordinieren.

Die zweite Alternative zu Vertrauen, Kontrolle im Sinne von Beaufsichtigung und Überprüfung, ist zunächst einmal unabhängig von Macht im oben definierten Sinn der Einflussnahme zu betrachten. Sie funktioniert anders als Machtausübung und Vertrauen nicht als Mittel zur Komplexitätsreduktion. Bei Kontrolle geht es im Gegenteil darum, sich die Komplexität der Arbeit des jeweiligen Mitarbeiters als Führungskraft durch Beaufsichtigung zu eigen zu machen, um auf diese Weise sicherzustellen, dass die eigenen
Erwartungen nicht enttäuscht werden. Aus ökonomischer Sicht hat Kontrolle somit im Vergleich zu Vertrauen immer den Nachteil der sehr hohen Transaktionskosten (vgl. Williamson 1993). Seine Mitarbeiter zu kontrollieren, ist deutlich aufwändiger, als ihnen zu vertrauen. Im Rahmen der Digitalisierung wird die Mitarbeiterkontrolle jedoch erheblich vereinfacht. So lassen sich z.B. bereits jetzt alle Schritte, die ein Mitarbeiter am Computer vollzieht, protokollieren und die Produktivität eines Mitarbeiters kann durch digitale Messstationen statistisch ausgewertet werden. Neben der Tatsache, dass solchen Kontrollen je nach Staat auch rechtliche Grenzen gesetzt sind, gibt es aber auch Bereiche, in denen Kontrolle gar nicht vollständig digital stattfinden kann. So sind z.B. die Erarbeitung eines neuen Konzeptes oder die Planung eines Projektes sowie alle anderen Tätigkeiten, die gedanklich zu komplex sind, um deren Qualität digital zu operationalisieren, nach wie vor nur durch die Führungskraft selbst zu kontrollieren.

In Anbetracht der oben stehenden Ausführungen ergibt sich der Schluss, dass Macht aufgrund der verflachenden Hierarchien an Bedeutung als Alternative zu Vertrauen verliert und Kontrolle aufgrund besserer Automatisierungsmöglichkeiten an Attraktivität gewinnt. Dennoch bleibt Vertrauen auch im Vergleich mit Kontrolle das attraktivere Konzept. Dies lässt sich dadurch begründen, dass Vertrauen im Gegensatz zu Kontrolle und Macht noch viele weitere positive Effekte auf Ebenen und Feldern hat, die für Organisationen im Allgemeinen und im Zuge der Digitalisierung eine wichtige Rolle spielen (vgl. Kap. 3. und Abschn. 3.1).

\section{Ergebnisse und praktische Implikationen}

Die vorangehende Analyse verdeutlicht eindrücklich, dass das Vertrauen zwischen Führungskraft und Mitarbeiter im Kontext der Digitalisierung nicht nur bedeutsam bleibt, sondern sogar noch bedeutsamer wird. Die Begründung hierfür liegt in einer Vermehrung der Anwendungsfelder sowie den Umständen, dass Macht als Alternativkonzept an Bedeutung verliert und auch digitale Kontrollmöglichkeiten Vertrauen gegebenenfalls ergänzen, aber nicht ersetzen können (vgl. Abb. 3).

Vertrauen ermöglicht die Bewältigung der Komplexität der vielfältigen neuen Arbeitsformen, indem es Führungskräfte und Mitarbeiter dazu befähigt, positive Erwartungen $\mathrm{zu}$ entwickeln und diese untereinander zu koordinieren. Vertrauen führt dazu, dass Führungskräfte und Mitarbeiter bereit sind, Risiken einzugehen und Unsicherheiten zu überwinden, die mit beständigen Veränderungen und der räumlichen und zeitlichen Ungebundenheit von Arbeit einhergehen. Die Nivellierung hierarchischer Unterschiede führt schließlich dazu, dass Macht als Alternativkonzept zu Vertrauen geschwächt wird. Auch digitale Kontrollmöglichkei- 
Abb. 3 Ergebnisse und praktische Implikationen

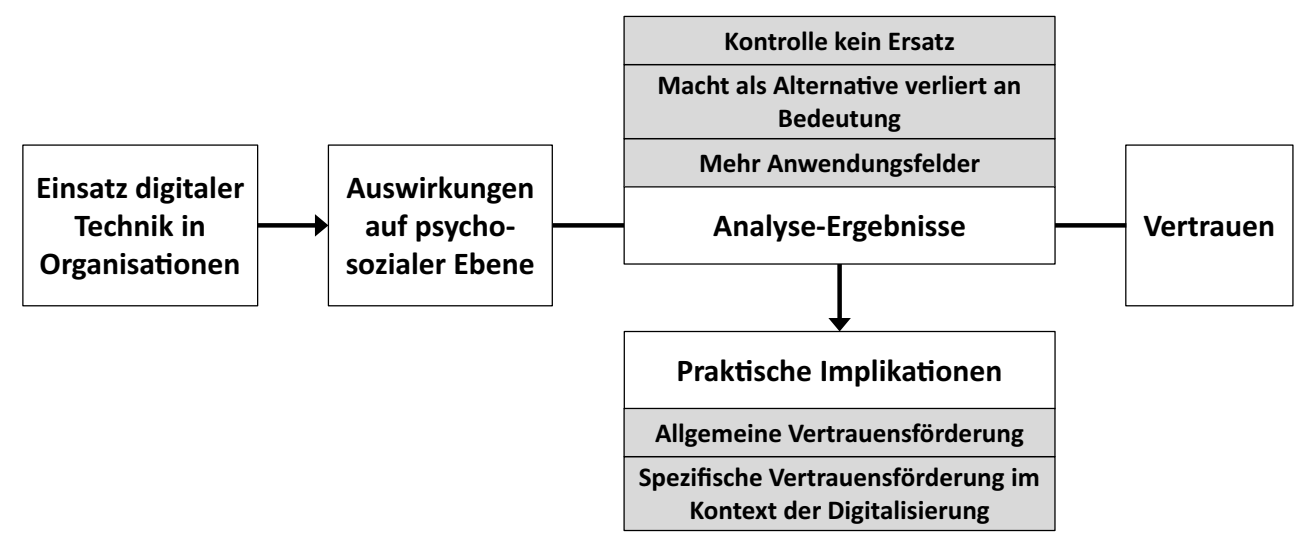

ten sind nicht in der Lage, Vertrauen zu ersetzen, da diese trotz vieler neuer Möglichkeiten und niedrigerer Transaktionskosten, einen eingeschränkten Anwendungsbereich haben und ein Fehlen der vielen weiteren positiven Auswirkungen von Vertrauen nicht kompensieren können. Somit wird deutlich, dass die Ausbildung von Vertrauensbeziehungen im Rahmen der digitalen Transformation eine lohnende Investition ist, die es mit gezielten Maßnahmen zu fördern gilt (vgl. Abb. 3).

Tab. 1 zeigt die auf den Ergebnissen dieses Beitrags basierenden Handlungsempfehlungen zur Förderung von Vertrauen im Überblick. Die in Tab. 1 genannten allgemeinen Empfehlungen werden zugunsten einer Ausführung der spezifischen Empfehlungen im Folgenden nicht weiter vertieft, da die generellen Bedingungen, die Vertrauensbeziehungen befördern, bereits in vielen Publikationen grundlegend beschrieben werden (vgl. z. B. Burke et al. 2007; Dirks und Ferrin 2002; Jäckel 2018; Mayer et al. 1995).

$\mathrm{Zu}$ den besonderen Herausforderungen der Digitalisierung zählt die räumliche und zeitliche Ungebundenheit von Arbeit, die dazu führt, dass Führungskraft und Mitarbeiter seltener Face-To-Face interagieren und somit auch weniger Gelegenheit zum Aufbau von Vertrauen bekommen (vgl. Abschn. 3.1). Um dieser Herausforderung zu begegnen, empfiehlt der vorliegende Beitrag zum einen, neue Mitarbeiter mit einem Trust-Boarding in das Unternehmen einzuführen, und zum anderen, das institutionen-basierte Vertrauen stärker zu fördern. Die im Folgenden skizzierte Idee eines Trust-Boardings leitet sich von dem Personalbereich gebräuchlichen Begriff des Onboardigs ab, der für die Einarbeitungsphase neuer Mitarbeiter steht (vgl. SpringerGabler 2018). Mit Trust-Boarding ist somit eine Einarbeitungsphase gemeint, die einen besonderen Fokus auf vertrauensbildende Maßnahmen legt. In dieser Phase hat die Führungskraft die Gelegenheit, dem Mitarbeiter zu vermitteln, dass sie kompetent, wohlwollend sowie integer und somit vertrauenswürdig ist (z. B. durch persönliche Gespräche, in denen der berufliche Werdegang, Wertvorstellungen sowie Perspektiven zur Personalentwicklung dargelegt wer- den). Nachhaltig gelingt dies natürlich nur, wenn die Führungskraft hierbei authentisch bleibt. Dann können Führungskraft und Mitarbeiter die spätere Arbeitsbeziehung, bei der virtualisierte Formen der Zusammenarbeit (z.B. Telekooperation) den Vertrauensaufbau erschweren, bereits auf einem höheren Vertrauenslevel starten und müssen das Vertrauen nicht erst auf die Entfernung hin entwickeln. Als ergänzende Maßnahme empfiehlt sich die Förderung des institutionen-basierten Vertrauens. Hiermit ist interpersonelles Vertrauen gemeint, bei dem die Vertrauenswürdigkeit einer Person wesentlich von der Organisation bzw. Institution abhängig ist, der diese Person durch den Vertrauensgeber zugerechnet wird (vgl. Zucker 1986). Eine Führungskraft, die einer Organisation angehört, die vertrauenserweckende Strukturen institutionalisiert hat, wird eher als vertrauenswürdig eingestuft, als eine Führungskraft, die einer Organisation angehört, die keine solchen oder sogar gegenteilige Strukturen institutionalisiert hat. Dies gilt insbesondere dann, wenn Führungskraft und Mitarbeiter sich selten persönlich begegnen, wie es durch räumliche und zeitliche Ungebundenheit von Arbeit immer häufiger der Fall ist. Konkret bedeutet dies, dass eine Organisation möglichst viele Strukturen schaffen sollte, die als kompetent, wohlwollend und integer wahrgenommen werden. Dazu gehören z. B. institutionalisierte Angebote der persönlichen und beruflichen Weiterentwicklung (z. B. Fortbildungen und gesicherte Aufstiegschancen), Angebote zur Verwirklichung von WorkLife-Balance (z.B. familienfreundliche Arbeitszeiten, betriebsinterne Kindergärten und Sportangebote), Mechanismen der Selbstkontrolle und -evaluation (z.B. 360-GradFeedback, Ideenmanagement bzw. Vorschlagswesen) sowie die Verankerung dieser Werte im organisationalen Leitbild und vieles mehr. Entscheidend für den Erfolg solcher organisationaler Strukturen ist, dass diese nicht nur formal existieren, sondern auch tatsächlich und verlässlich gelebt werden. Nur so kann die Attribuierung von Vertrauenswürdigkeit langfristig Bestand haben.

Der letzte Aspekt, über den im Rahmen der praktischen Implikationen gesprochen werden muss, betrifft die durch 
Tab. 1 Allgemeine und spezifische Handlungsempfehlungen im Überblick

\begin{tabular}{|c|c|}
\hline & $\begin{array}{l}\text { Handlungsempfehlungen zur Förderung von } \\
\text { Vertrauen }\end{array}$ \\
\hline \multirow[t]{5}{*}{ Allgemein } & $\begin{array}{l}\text { Stärkung der Vertrauenswürdigkeit der Führungs- } \\
\text { kraft }\end{array}$ \\
\hline & Vertrauensvorschuss durch die Führungskraft \\
\hline & Delegation relevanter Aufgaben an Mitarbeiter \\
\hline & $\begin{array}{l}\text { Förderung der Selbstwirksamkeitserfahrung des } \\
\text { Mitarbeiters }\end{array}$ \\
\hline & Systemisches Denken und Handeln \\
\hline \multirow{3}{*}{$\begin{array}{l}\text { Spezifisch } \\
\text { im Digita- } \\
\text { lisierungs- } \\
\text { Kontext }\end{array}$} & Trust-Boarding (erster Kontakt) \\
\hline & $\begin{array}{l}\text { Stärkung des institutionen-basierten Vertrauens (auf } \\
\text { organisationaler Ebene) }\end{array}$ \\
\hline & Gezielter Einsatz neuer Formen digitaler Kontrolle \\
\hline
\end{tabular}

Digitalisierung neu ermöglichten Kontrollformen. Denn es scheint evident, dass in manchen Bereichen Vertrauen nicht mehr notwendig ist, da die zu bewältigende Unsicherheit durch aus digitaler Kontrolle gewonnenem Wissen effizient beseitigt werden kann. So kann z. B. die Anwesenheit eines Mitarbeiters bzw. sein Standort auf verschiedene Weise digital erfasst und verfolgt werden. Sowohl aus ethischer Perspektive als auch aus ökonomischer Perspektive ist jedoch Vorsicht geboten und genau zu analysieren, ob tatsächlich alle Kontrollen, die digital ermöglicht werden, auch tatsächlich durchgeführt werden sollten. Kontrollen sollten nur dann eingesetzt werden, wenn sie das Vertrauen zwischen Führungskraft und Mitarbeiter nicht stören. Denn wie oben ausgeführt, hat Vertrauen vielfältige positive Effekte, die nicht durch die aus Kontrolle entstandene Gewissheit in einem begrenzten Bereich aufgewogen werden können (vgl. Kap. 3). Ein Mitarbeiter, der das Gefühl hat, dass seine Arbeit kontrolliert wird, weil ihm nicht vertraut wird, wird weniger zufrieden mit seiner Arbeit sein, sich unsicherer fühlen, mehr negativen Stress haben, weniger Wissen teilen und letztlich auch weniger produktiv sein. Dennoch gibt es auch im Rahmen einer Vertrauensbeziehung Möglichkeiten für Kontrolle. Kontrollen können insbesondere dann mit Vertrauen einhergehen, wenn sie nicht einseitig von der Führungskraft genutzt werden. Geeignet für die Vertrauensbeziehung zwischen Führungskraft und Mitarbeiter sind daher z.B. digitale Kontrollen, die der Selbstkontrolle des Mitarbeiters dienen und deren Daten nur für den Mitarbeiter bestimmt sind. Ein noch größeres Potenzial für die Vertrauensbildung ist zu erwarten, wenn Kontrollen von Mitarbeiter und Führungskraft zusammen durchgeführt und evaluiert werden, um darauf aufbauend gemeinsame Optimierungsvorschläge abzuleiten.

\section{Ausblick}

Die Digitalisierung der Arbeitswelt hat begonnen und wird sich mit der voranschreitenden technischen Entwicklung immer weiter entwickeln. Die oben stehenden Ausführungen verdeutlichen, dass Vertrauen den Prozess der Digitalisierung an vielen Stellen positiv unterstützen kann und somit in Zukunft noch bedeutsamer für ein erfolgreiches organisationalen Handeln wird. Der vorliegende Beitrag beschränkt sich dabei bewusst auf ein konzeptionelles Aufzeigen dieses Potenzials und möglicher Erklärungen in Bezug auf die Wirkzusammenhänge zwischen Vertrauen und den einzelnen Aspekten der Digitalisierung. In diesem Sinne füllt er eine Lücke zwischen vielfachen oberflächlichen Bezügen und Studien, die sich lediglich auf einen spezifischen Teilaspekt beziehen. Der Beitrag schafft so eine thematische Grundlegung, die neue Perspektiven für weiterführende empirische Studien eröffnet. Hierbei bieten sich sowohl quantitative als auch qualitative Studien an, die den Zusammenhang zwischen Vertrauen und spezifischen digitalen Phänomenen im organisationalen Kontext differenzierter betrachten wollen, als dies bislang häufig der Fall ist; z. B. zu den Themen Automatisierung, digitale Organisationsstrukturen und -kultur, digitales Lernen, Controllingund Trackingsysteme u.v. m. Abschließend verdeutlicht der Beitrag, dass zukünftige Führungsmodelle im Kontext der digitalen Transformation Vertrauen noch stärker als bisher miteinbeziehen müssen. Die aus den Erkenntnissen abgeleiteten praktischen Implikationen sollen dabei helfen, eine Führung zu entwickeln, die erfolgreich Vertrauensbeziehungen zu den Mitarbeitern etabliert und gleichzeitig neugewonnene Möglichkeiten digitaler Kontrolle integriert.

Funding Open Access funding provided by Projekt DEAL.

Open Access Dieser Artikel wird unter der Creative Commons Namensnennung 4.0 International Lizenz veröffentlicht, welche die Nutzung, Vervielfältigung, Bearbeitung, Verbreitung und Wiedergabe in jeglichem Medium und Format erlaubt, sofern Sie den/die ursprünglichen Autor(en) und die Quelle ordnungsgemäß nennen, einen Link zur Creative Commons Lizenz beifügen und angeben, ob Änderungen vorgenommen wurden.

Die in diesem Artikel enthaltenen Bilder und sonstiges Drittmaterial unterliegen ebenfalls der genannten Creative Commons Lizenz, sofern sich aus der Abbildungslegende nichts anderes ergibt. Sofern das betreffende Material nicht unter der genannten Creative Commons Lizenz steht und die betreffende Handlung nicht nach gesetzlichen Vorschriften erlaubt ist, ist für die oben aufgeführten Weiterverwendungen des Materials die Einwilligung des jeweiligen Rechteinhabers einzuholen.

Weitere Details zur Lizenz entnehmen Sie bitte der Lizenzinformation auf http://creativecommons.org/licenses/by/4.0/deed.de. 


\section{Literatur}

Ahlers, E. (2018). Die Digitalisierung der Arbeit. Verbreitung und Einschätzung aus Sicht der Betriebsräte. WSI Report. Düsseldorf: Hans-Böckler-Stiftung.

von Ameln, F., \& Kramer, J. (2012). Macht und Führung. Gruppendynamik und Organisationsberatung, 43(2), 189-204.

Bachmann, R. (2003). Trust and power as means of coordinating the internal relations of the organization: A conceptual framework. In B. Nooteboom \& F. Six (Hrsg.), The trust process in organizations: Empirical studies of the determinants and the process of trust development (S. 58-74). Cheltenham: Edward Elgar.

Bruckner, L., \& Werther, S. (2018). Allgemeiner Überblick über Arbeit 4.0. In I. S. Werther \& L. Bruckner (Hrsg.), Arbeit 4.0 aktiv gestalten (S. 15-22). Berlin: Springer.

Bundesministerium für Arbeit und Soziales. (2016). Weißbuch Arbeiten 4.0. https://www.bmas.de/SharedDocs/Downloads/DE/PDFPublikationen/a883-weissbuch.pdf. Zugegriffen: 11. Okt. 2019.

Burke, C.S., Sims, D.E., Lazzara, E.H., \& Salas, E. (2007). Trust in leadership: A multi-level review and integration. The Leadership Quarterly, 18(6), 606-632.

Depiereux, P., \& van Alphen, C. (2018). Führung im Zeitalter der Digitalisierung - eine Standortbestimmung. In S. Werther \& L. Bruckner (Hrsg.), Arbeit 4.0 aktiv gestalten (S. 29-36). Berlin: Springer.

Deutsche Gesellschaft für Personalführung e. V. (2016). Leitfaden: Führen im digitalisierten Unternehmen. https://www.dgfp. de/fileadmin/user_upload/DGFP_e.V/Medien/Publikationen/ Praxispapiere/201603_Praxispapier_Fuehren-im-digitalisiertenUnternehmen.pdf. Zugegriffen: 8. Okt. 2019.

Diekhöner, P. K. (2018). The Trust Economy. Warum jedes Unternehmen eine Vertauensstrategie braucht, um im digitalen Zeitalter zu überleben. Berlin: Springer Gabler.

Dirks, K. T., \& Ferrin, D.L. (2002). Trust in leadership: meta-analytic findings and implications for research and practice. Journal of applied psychology, 87(4), 611.

Endress, M. (2002). Vertrauen. Bielefeld: Transkript.

Gargiulo, M., \& Ertug, G. (2006). The dark side of trust. In R. Bachmann \& A. Zaheer (Hrsg.), Handbook of Trust Research (S. 165-186). Cheltenham: Edward Elgar.

Geramanis, O. (2001). Vertrauen und Vertrauensspielräume in Zeiten der Unkontrollierbarkeit. Doctoral dissertation. München: Universtät der Bundeswehr München, Universitätsbibliothek.

Hirsch-Kreinsen, H. (2015). Digitalisierung von Arbeit: Folgen, Grenzen und Perspektiven. Soziologisches Arbeitspapier, 43. Dortmund: Wirtschafts- und Sozialwissenschaftliche Fakultät - Technische Universität.

Hoe, S. L. (2007). Is interpersonal trust a necessary condition for organisational learning? Journal of Organisational Transformation \& Social Change, 4(2), 149-156.

Jäckel, A. (2018). Gesundes Vertrauen in Organisationen. Eine empirische Untersuchung der Vertrauensbeziehung zwischen Führungskraft und Mitarbeiter. Wiesbaden: Springer.

Kauffeld, S., Handke, L., \& Straube, J. (2016). Verteilt und doch verbunden: Virtuelle Teamarbeit. Gruppe. Interaktion. Organisation. Zeitschrift für Angewandte Organisationspsychologie (GIO), 47(1), 43-51.

Kruse, P., \& Schomburg, F. (2016). Führung im Wandel: Ohne Paradigmenwechsel wird es nicht gehen. In O. Geramanis \& K. Hermann (Hrsg.), Führen in ungewissen Zeiten (S. 3-16). Wiesbaden: Springer.

Läsker, K. (2019). „Der Chip ist Teil meines Körpers geworden“. Spiegel Online. https://www.spiegel.de/karriere/schweden-tuimitarbeiter-tragen-mikrochips-unter-der-haut-a-1287060.html. Zugegriffen: 22. Okt. 2019.

Luhmann, N. (2000). Vertrauen. Stuttgart: Lucius \& Lucius.

Malczok, M., \& Kirchhoff, S. (2019). Digitalisierung und Partizipation - Brauchen wir ein neues Skill Set für Führungskräf- te? In M. Stumpf (Hrsg.), Digitalisierung und Kommunikation (S. 211-229). Wiesbaden: Springer.

Mayer, R. C., Davis, J.H., \& Schoorman, F.D. (1995). An integrative model of organizational trust. Academy of management review, 20(3), 709-734.

Molina-Morales, F.X., Martínez-Fernández, M.T., \& Torlo, V.J. (2011). The dark side of trust: the benefits, costs and optimal levels of trust for innovation performance. Long Range Planning, 44(2), 118-133.

Möllering, G. (2001). The nature of trust: from Georg Simmel to a theory of expectation, interpretation and suspension. Sociology, 35(2), 403-420.

Möllering, G., Bachmann, R., \& Lee, H. S. (2004). Introduction: Understanding organizational trust-foundations, constellations, and issues of operationalisation. Journal of Managerial Psychology, 19(6), 556-570.

Raven, B.H., Schwarzwald, J., \& Koslowsky, M. (1998). Conceptualizing and Measuring a Power/Interaction Model of Interpersonal Influence. Journal of Applied Social Psychology, 28(4), 307-332.

Schaff, A. (2019). Arbeit 4.0. Risiken für die psychische Gesundheit. In B. Hermeier, T. Heupel \& S. Fichtner-Rosada (Hrsg.), Arbeitswelten der Zukunft (S. 303-322). Wiesbaden: Springer.

Schmiech, C. (2018). Der Weg zur Industrie 4.0 für den Mittelstand. In D. Wolff \& R. Göbel (Hrsg.), Digitalsierung: Segen oder Fluch (S. 1-28). Berlin: Springer.

Schönfelder, C. (2019). Digitale Kommunikation und Führung 4.0 - zum Potenzial neuer Kommunikationsinstrumente für aktuelle Führungsrollen. In M. Stumpf (Hrsg.), Digitalisierung und Kommunikation (S. 199-210). Wiesbaden: Springer.

Skinner, D., Dietz, G., \& Weibel, A. (2014). The dark side of trust: when trust becomes a "poisoned chalice". Organization., 21(2), 206-224.

Springer Gabler (2018). Gabler Wirtschaftslexikon. Stichwort: Arbeitszufriedenheit. https://wirtschaftslexikon.gabler.de/definition/ personalbeschaffung-44990/version-268291. Zugegriffen: 15 . Okt. 2019.

Werther, S., Bruckner, L., \& Mann, F. (2018). Psychologische Perspektiven auf Arbeit 4.0. In S. Werther \& L. Bruckner (Hrsg.), Arbeit 4.0 aktiv gestalten (S. 48-55). Berlin: Springer.

Williamson, O.E. (1993). Calculativeness, trust, and economic organization. The Journal of Law \& Economics, 36(1), 453-486.

Zucker, L. G. (1986). Production of trust: Institutional sources of economic structure. In B. M. Staw \& L. L. Cummings (Hrsg.), Research in Organizational Behavior (Bd. 8, S. 53-111). Greenwich: JAI Press.

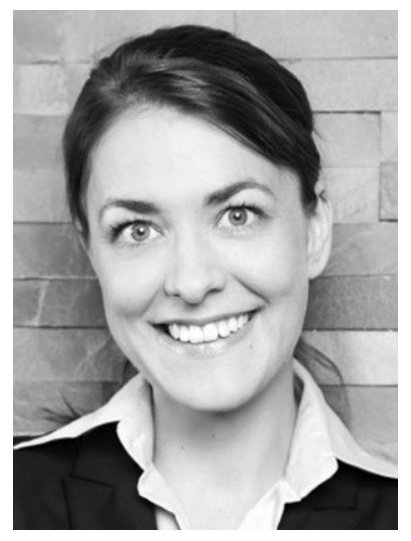

Dr. Ariane Jäckel ist Dozentin an verschiedenen Universitäten/ Hochschulen und Weiterbildungsinstituten. Seit 2007 ist sie als Beraterin und Coach tätig. Sie studierte Wirtschaftswissenschaften und Wirtschaftspädagogik an der Universität Kassel und der Corvinus University of Budapest und promovierte am Fachgebiet Arbeitsund Organisationspsychologie der Universität Kassel. Im Rahmen ihrer wissenschaftlichen Arbeit leitete sie unterschiedliche Praxisprojekte mit großen Industrieunternehmen und Non-Profit-Organisationen. Ihr derzeitiges Forschungsinteresse umfasst die Themengebiete Lernen, Führung, Vertrauen und Veränderungsmanagement. 